\title{
Zur Frage nach der chemischen Zusammensetzung der Bakterienkörper des Azotobacter chroococcum.
}

\author{
Von
}

W. L. Omeliansky und N. O. Sieber.

(Aus den mikrobiologischen und chemischen Abteilungen des Kaiserlichen Instituts für experimentelle Medizin zu St. Petersburg.)

(Der Redaktion zugegangen am 14. November 1913.)

Das Studium der chemischen Vorgänge in Bakterien bietet von verschiedenen Standpunkten aus bedeutendes Interesse. Berücksichtigung finden jedoch vor allem die plastische Tätigkeit der Mikroben einerseits und die durch dieselben bedingten Dessimilationsprozesse anderseits. Die Chemie der Bakterienzelle ist in dieser letzteren Beziehung mehr oder weniger eingehend studiert und die kosmische Bedeutung der Mikroben, als wirkenden Prinzipien der Zersetzung und Mineralisation von organischer Substanz ist aufgeklärt.

Nicht geringeres Interesse bieten auch diejenigen synthetischen Prozesse, welche der plastischen Mikrobentätigkeit zugrunde liegen. Diese Prozesse verlaufen bei verschiedenen Bakterien verschieden.

Bei denjenigen, zu deren Ernährung komplizierte organische Verbindungen dienen, geht der Synthese eine Zersetzung organischer Substanz unter Bildung einfachster Produkte, welche als Ausgangsmaterial zum Aufbau des Körpers dienen, voraus. Leider bleibt es bis jetzt unaufgeklärt, welcher Art diese Endzersetzungsprodukte sind, und deshalb ist das Verständnis des Mechanismus der synthetischen Prozesse unmöglich.

Bedeutende Vorzüge bietet das Studium der plastischen Tätigkeit prototropher Bakterien, welche ihr Eiweiß aus einfachsten, , rein mineralischen Stoffen, wie Kohlensäure, freiem

Stickstoff, salpetersauren Salzen u. d. m., aufbauen. Kennt man 
die Ausgangs- und Endprodukte, so kann man sich auch einen mehr oder weniger klaren Begriff von dem gesamten synthetischen Prozesse, von der Schaffungssynthese dieser Bakterien bilden.

Von bedeutendem theoretischen Interesse ist die Eiweißsynthese durch stickstofffixierende Bakterien auf Kosten des freien atmosphärischen Stickstoffs. Aus welchen Phasen setzt sich dieser Prozeß zusammen und in welcher Anordnung folgen dieselben aufeinander? Von verschiedenen Autoren sind hierüber verschiedene Voraussetzungen geäußert, welche doch alle einen mehr oder weniger hypothetischen Charakter haben. Jedenfalls unterliegt es keinem Zweifel, daß unter Einwirkung der stickstofffixierenden Bakterien Aktivierung des elementaren Stickstoffs stattfindet, welcher hierbei die Fähigkeit gewinnt, mit anderen Elementen in Verbindung zu treten.

Gautier und Drouin ${ }^{1}$ ) nahmen an, daß die Bindung von Stickstoff durch Bakterien vermittelst seiner Oxydation unter Einwirkung von lebendem Protoplasma in ähnlicher Weise vor sich geht, wie Stickstoff bindung in feuchter Luft bei Oxydation von organischer Substanz zu beobachten ist. Dieselbe Ansicht vertrat auch Berthelot. ${ }^{2}$ )

S. Winogradsky, ${ }^{3}$ ) welcher als erster die anaërobe stickstoffixierende Art Clostridium Pasteurianum in Reinkultur erhielt und deren Eigenschaften studierte, äußerte die Voraussetzung, daß die Fixation von Stickstoff durch dieses Bakterium «comme l'effet de la rencontre de l'azote gazeux et de l'hydrogène naissant au sein du protoplasma vivant» betrachtet werden könne; weiter sagt er: «il est permis de supposer, que la synthèse de l'ammoniaque pourrait en être le résultat immédiat.» Diese Hypothese reicht bereits in die Zeit .Saussures4) zurück, welcher im Jahre 1833 einer ähnlichen Meinung über die bei Gärung organischer Stoffe zu beobachtende

1) Gautier et Drouin, Comptes rendus des séances de l'Académie des sciences, Paris, t. 106, 113 et 114.

2) Berthelot, Comptes rendus, t. 116.

s) S. Winogradsky, Comptes rendus, t. 116 et 118.

4) Saussure, Mém. de la soc. de phys. et d'hist. nat. Genève, t. 6, p. 560 (1833). 
Stickstoff bindung geäußert hat. Später schlossen sich derselben Anschauung Browne, ${ }^{1}$ ) Mulder, ${ }^{2}$ ) Simon, $\left.{ }^{3}\right)$ Reinke ${ }^{4}$ ) u. a. an.

Im Jahre 1901 wurde von. Beijerinck ${ }^{j}$ ) aus dem Boden (in Delft) einè äërobe stickstofffixierende Art - Azotobacter chroococcum, welche in der Natur ebenso weit verbreitet ist, wie das Clostridium Pasteurianum, isoliert. $\mathrm{Zu}$ seiner .ersten Veröffentlichung hat Beijerinck dieser Art die Fähigkeit zugeschrieben, den freien Stickstoff der Atmosphäre in salpetersaure Salze umzuwandeln, später jedoch gab er diese Ansicht auf. In einer in Gemeinschaft mit van Delden ${ }^{6}$ ) veröffentlichten Arbeit schrieb Beijerinck die Stickstofffixation nicht dem Azotobacter, sondern anderen Arten zu, welche den Gattungen Granulobacter, Radiobacter und Aerobacter angehören. Diese Bakterien binden, indem sie in Gemeinschaft mit Azotobacter wachsen, den freien Stickstoff und lassen hierbei eine lösbare Verbindung (nicht eiweißartiger Natur) entstehen, welche in die Nährlösung übergeht und für den Azoto-bacter als Stickstoffnahrung dient. Jedoch konnte die Anwesenheit dieser hypothetischen Substanz in dem Nährboden von den Autoren nicht nachgewiesen werden. Es lag die Voraussetzung nahe, daß sie unmittelbar nach ihrer Entstehung vom Azotobacter ausgenutzt werde. Hierbei blieb jedoch der Umstand unerklärlich, weshalb diese für den Azotobacter so nahrhafte Substanz für die Ernährung anderer Arten vollständig unbrauchbar ist. Überhaupt ist ihre chemische Natur scbließlich unbekannt geblieben, und selbst ihre Existenz muß als zweifelhaft anerkannt werden. Beijerincks Hypothese wurde von späteren Autoren, die dem Azotobacter als Stickstoffixator in vollem Maße wieder zu seinem guten Namen verhalfen, widerlegt.

1) Browne, The American Muck Book, 1858.

2) Mulder, Chemie der Ackerkrume, 1863.

3) Simon, Landw. Vers.-Stat., Bd. 18, S. 454 (1875).

4) Reinke, Ber. d. deutsch. bot. Ges., Bd. 21, S. 379 (1903).

5) Beijerinck, Centralbl. f. Bakt., 2. Abt., Bd. 7, S. 561 (1901).

- Beijerinck und van Delden, Centralbl. f. Bakt., 2. Abt., Bd. 9, S. 3 (1902). 
Als erste sprachen sich Gerlach und Vogel') gegen Beijerincks Hypothese aus; ihrer Meinung nach werden bei der Sticktofffixikation im Körper von Azotobacter durch Angliederung von Stickstoff an die stickstofffreie organische Substanz Amidoverbindungen gebildet, später aber aus den Amidoverbindungen Eiweiß aufgebaut. Lösliche Stickstoffverbindungen können hierbei nicht nachgewiesen werden. Sie können zuweilen nur in Mischkulturen von Azotobacter mit anderen Arten, als Ergebnis einer sukzessiven Zersetzung der früher entstandenen Eiweißstoffe, nachgewiesen werden.

Nach neueren Angaben von Krzemieniewski²) gehen ca. 13,3\% des durch Azotobacter gebundenen Stickstoffs in Lösung über. Es ist jedoch möglich, daß diese Substanzen nicht ein primäres Assimilationsprodukt darstellen, sondern infolge von Zersetzung und Autolyse abgestorbener Zellen des Azotobacter gebildet werden. Dieselbe Erwägung hat auch auf die Angaben von Lipman, ${ }^{3}$ ) welcher nicht nur im Filtrat einer abgetöteten Azotobacterkultur nach Fällung mit Phosphorwolframsäure, sondern auch unmittelbar in einer durch Chamberland-Filter filtrierten Kultur Stickstoff nachweisen konnte, Bezug.

Auch nach der Ansicht von Heinze ${ }^{4}$ ) werden Amidoverbindungen in den Zellen von Azotobacter durch direkte Angliederung von freiem Stickstoff, aller Wahrscheinlichkeit nach an Kohlenhydrate, gebildet, wobei als Initialprodukt dieser Angliederung Salze der Carbaminsäure entstehen, während erst später aus ihnen Amidosäuren und Eiweißsubstanzen hervorgehen.

Stoklasa, ) welcher wahrscheinlich mit einer verunreinigten Azotobacterkultur $\mathrm{zu}$ tun hatte und Ausscheidung von freiem Wasserstoff beobachten konnte, schrieb diesem letzteren für die.Stickstoffassimilation durch die betreffende Bakterienart eine große Bedeutung zu. In einer späteren Ver-

1) Gerlach u. Vogel, Centralbl. f. Bakt., 2. Abt., Bd. 9, S. 884 (1902).

2) Krzemieniewski, Bull. intern. de l'Ac. des Sc. de Cracovie, 1908.

3) Lipman, N. Jersey St. Rep., t. 25, 1904; t. 26, 1905.

4) Heinze, Centralbl. f. Bakt., 2. Abt., Bd. 12, 1904.

5) Stoklasa, Centralbl. f. Bakt., 2. Abt., Bd. 21, 1908. 
öffentlichung ${ }^{1}$ ) stellt er die Hypothese auf, daß Salze der Blausäure als Zwischenprodukt bei der Stickstofffixation gebildet werden. «Der Wasserstoff», schreibt Stoklas a (S. 626), «welcher bei den Stoffwechselprozessen des Azotobacter entsteht, hat sicherlich in statu nascendi eine bestimmte Aufgabe bei der Bindung des elementaren Stickstoffs. Wie die Eiweißsynthese verläuft, das ist uns heute noch sehr schwer zu sagen, da wir unsere Versuche noch nicht beendet haben; allein, wie es uns nach allem scheint, entsteht als erstes Produkt etwa Cyanwasserstoff, welcher sodann die Grundlage der Eiweißsynthese bei dem weiteren Stoffwechselprozesse abgibt. Cyanwasserstoff wurde in der Pflanzenzelle tatsächlich schon nachgewiesen, und es ist uns auch bereits gelungen, in gewissen Fällen den Cyanwasserstoff unter bestimmten Kauteln in zerrissenen Zellen der jungen Kultur von Azotobacter nachzuweisen.) Ohne die Wahrscheinlichkeit dieser Hypthese weiter zu beurteilen, wollen wir hier nur bemerken, daß schon Stoklasas Grundprämisse in betreff der Wasserstoffausscheidung durch Azotobacterkulturen nicht richtig ist, denn dieses Gas wird durch Azotobacterkulturen niemals ausgeschieden. Und deswegen können die Erwägungen, welche zur Erklärung des Mechanismus der Stickstofffixation durch das anaërobe Glostridium Pasteurianum von Geltung sind, hier durchaus keine Verwendung finden.

Schlieblich wollen wir noch erwähnen, daß nach Lipmans Ansicht (loc. cit.) als erstes Produkt der Stickstoffassimilation durch das Azotobacter eine Aminosäure, am allerwahrscheinlichsten Alanin, nach Loew und $\mathrm{Aso}^{2}$ ) aber salpetrigsaures Ammoniak $\left(\mathrm{N}_{2}+2 \mathrm{H}_{2} \mathrm{O}=\mathrm{NH}_{4} \mathrm{NO}_{2}\right)$ auftritt, welches später $\mathrm{zu}$ Ammoniak, das zum Eiweißaufbau dient, reduziert wird.

Die eben angeführte Übersicht der Literaturangaben beweist uns, wie weit die Meinungen verschiedener Autoren über den Mechanismus der Stickstoffixation durch Bakterien und deren Zürischenprodukte auseinandergehen und wie hypothetisch die Voraussetzungen der Autoren sind.

1) Stoklasa, Centralbl. f. Bakt., 2. Abt., Bd. 21, 1908.

2) Loew und Aso, Centralbl. f. Bakt., 2. Abl., Bd. 22, 1909. 
Zahlreichere tatsächliche Hinweise gibt uns die chemische Analyse der Körper von stickstofffixierenden Bakterien. In Anbetracht des eigenartigen Typus der Stickstoffernährung dieser Mikroorganismen gewinnen die Befunde ein besonderes Interesse. Es drängen sich hier eine Reihe von Fragen, welche für die Charakteristik dieser interessanten Gruppe von Mikroorganismen von wesentlicher Bedeutung sind, auf. Die erste von diesen wäre, wie reich an Stickstoff das Eiweiß dieser Bakterien ist und ob sie sich in dieser Hinsicht von den gewöhnlichen Saprophyten, welche zu ihrer Ernährung des gebundenen Stickstoffs bedürfen, unterscheiden? Weiter fragt sich, ob der Prozentgehalt an Stickstoff sich in ihrem Körper in Abhängigkeit von den Kulturbedingungen, den Rasseneigenheiten, dem Alter des Aussaatmaterials usw. verändert. Schließlich wäre $\mathrm{zu}$ entscheiden, welcher Natur die hydrolytischen Produkte der stickstofffixierenden Bakterien sind und ob dieselben einen Unterschied von denjenigen gewöhnlicher Bakterien bieten.

Indem wir alle diese Fragen aufwerfen, machen wir auf eine endgültige Lösung derselben durchaus keinen Anspruch. Unsere Untersuchungen sollen nur die ersten Schritte auf diesem Gebiete sein.

Als Material $\mathrm{zu}$ unseren Untersuchungen wählten wir eine Kultur des Azotobacter chroococcum, da von dieser Art im Vergleich zum anaëroben Clostridium Past. leichter Massenkulturen zu gewinnen und eine erforderliche Menge des Materials zu beschaffen ist. Zur Züchtung des Azotobacter chroococcum wurde ein fester Nährboden von folgender $\mathrm{Zu}$ sammensetzung verwandt:

$\begin{array}{ll}\text { Dextrin }{ }^{1} \text { ) } & 2,0 \mathrm{~g} \\ \text { Kaliumphosphat } & 0,1 \\ \text { Magnesiumsulfat } & 0,05 \\ \text { Calciumcarbonat } & 1,0 \\ \text { Agar agar } & 2,0 \\ \text { Destilliertes Wasser } & 100\end{array}$

1) Das zu diesen Versuchen verwandte Dextrin zeigte mit Jod eine dunkelviolette Färbung und reduzierte energisch Fehling sche Lösnug. 
Dieser Agarnährboden wurde in Petrischalen, deren Durchmesser $15 \mathrm{~cm}$ betrug, verteilt (ca. $40 \mathrm{ccm}$ auf jede Schale). Auf die Oberfläche des erstarrten Agars brachten wir einige Tropfen einer Emulsion der Azotobacterkultur, welche mit einem in stumpfem Winkel gebogenen Glasstäbchen über die ganze Agarfläche verteilt wurden. $\mathrm{Zu}$ unseren Versuchen diente eine Kultur des Azotobacter chroococcum, welche aus einer Erdbodensorte Wolhyniens isoliert wurde. Der Stamm war sehr aktiv und entwickelte im Laufe mehrerer Tage auf dem erwähnten Nährboden das dunkelbraune Pigment.

Die besäten Petrischalen wurden in den Thermostaten bei $30^{\circ}$ gestellt. Bereits nach Verlauf von 2-3 Tagen bedeckte sich die Agarfläche mit einer gallertigen Azo tobactermasse, welche allmählich eine dunkelbraune Färbung annahm. Am sechsten Tage war die ganze Plattenoberfläche gewöhnlich mit einer dunkelbraunen, zuweilen fast schwarzen Masse von Azotobacterkörpern bedeckt, die wir mit Hilfe eines Objektträgers mit geschliffenen Rändern sammelten. Die auf diese Weise gewonnene dunkelbraune, schleimige Bakterienmasse verdünnten wir mit einer geringen Menge destillierten Wassers und filtrierten sie durch Glaswolle, um die Partikeln des Agarnährbodens, welche bei Entfernung des Bakterienbelages etwa mit abgeschabt werden konnten, zu entfernen. Um das Filtrat gegen Zersetzung zu schützen, wurde es möglichst rasch im Vakuum bei $37^{\circ}$ getrocknet. Unter diesen Bedingungen fand jedoch rasch Säuerung der Bakterienmasse statt: es trat Buttersäuregärung mit ausgiebiger Gasentwickelung ein. Aus diesem Grunde mußte in weiteren Versuchen dieses Verfahren aufgegeben und folgendes benutzt werden. Die teerartige Masse der Azotobacterzellen wurde in dünner Schicht in Krystallisationsschälchen, welche auf den kupfernen Boden eines auf $37^{\circ}$ temperierten Brutschrankes zu stehen kam, gegossen. Unter diesen Bedingungen trat gewöhnlich keine Butterssäuregärung ein. Die getrocknete Masse der Azotobacterkörper bildete dunkelbraune, hornartige Stücke, welche sich leicht von dem Glasboden des Schälchens ablösen ließen. Diese Stücke wurden zu einem feinsten Pulver zerrieben, welches dann zur Analyse 
diente. Von einer weiteren Reinigung der Bakterienkörper vermittelst der Dialyse sahen wir $a b$, weil wir befürchteten, daß sie irgend welcher durch die Membran dringender Bestandteile verlustig werden könnten. Die zerkleinerte trockene Masse der Az o tobacterkörper stellte ein sehr leichtes, dunkelbraunes Pulver, welches seinem Aussehen nach an getrocknete und durch ein sehr feines Sieb gelassene Schwarzerde erinnerte, dar. Wurden die beiden Pulver in zwei nebeneinander stehende Gefäße geschültet, so waren sie durchaus nicht voneinander zu unterscheiden.

$1 \mathrm{~g}$ der trockenen Bakterienmasse entsprach dem Inhalte von 8-9 Petrischalen. Indem wir Massenkulturen des Azotobacter anlegten, konnten wir schließlich ungefähr $500 \mathrm{~g}$ der trockenen Bakterienmasse, welche wir zur Analyse verwandten, zusammenbringen.

Die einzelnen Untersuchungen ergaben folgende Resultate:

1.Die Bestimmung des Wassergehalts wurde durch Trocknen bei $100-105^{\circ}$ bis zum konstanten Gewicht in drei Portionen des Materials ${ }^{1}$ ) vorgenommen und ergab folgendes:

\begin{tabular}{|c|c|c|c|}
\hline & $\mathbf{A}$ & B & C \\
\hline $\begin{array}{l}\text { Gewicht in Grammen des luft- } \\
\text { trockenen Materials . . }\end{array}$ & 0,5042 & 0,6896 & 1,025 \\
\hline $\begin{array}{l}\text { Gewichtsverlust beim Trocknen } \\
\text { in Grammen . . . . . }\end{array}$ & 0,0379 & 0,0432 & 0,0632 \\
\hline $\begin{array}{c}\text { Prozentgehalt des Wassers im } \\
\text { lufttrockenen Material . . . }\end{array}$ & 7,51 & 6,26 & 6,12 \\
\hline
\end{tabular}

Drei Bestimmungen ergaben im Durchschnitt $-6,63 \%$.

Es ist möglich, daß es sich bei der Bestimmung des Wassergehalts auch um Verlust an flüchtigen Substanzen handeln kann.

2. Bestimmung des Aschegehaltes in 3 Proben:

\begin{tabular}{l|l|l|l}
\hline & \multicolumn{1}{|c|}{ A } & \multicolumn{1}{|c|}{ B } & \multicolumn{1}{|c}{ C } \\
\hline Gewicht in Grammen . . . . . . & 0,9985 & \multicolumn{1}{|c|}{1,2629} & 0,2467 \\
Gewicht der Asche in Grammen . & 0,0453 & 0,0522 & 0,0064 \\
Prozentgehalt der Asche . . . . & 4,54 & 4,11 & 3,83
\end{tabular}

1) Diese 3 Portionen gehörten verschiedenem Materiale, welches zu verschiedenen Zeiten gesammelt war. Unter den Bezeichnungen A, B und C sind die einzelnen Portionen auch in den übrigen-Analysen verzeichnet. 
Drei Bestimmungen ergaben im Durchschnitt 4,16\%. Dieser Wert hat durchaus keine absolute Bedeutung, da der Aschegehalt in Bakterien nach betreffenden Literaturangaben von dem Gehalte’ an Mineralstoffen im Nährboden abhängig ist.

Was den Bestand der Ascheelemente anbetrifft, so besteht nach Angaben von Stocklasa ${ }^{1}$ ) die Asche von Azotobacter im wesentlichen aus $\mathrm{K}_{2} \mathrm{O}$ und $\mathrm{P}_{2} \mathrm{O}_{5}$. Durch ihre Gegenwart wird die Oxydationstätigkeit des Azotobacter, zugleich auch die Stickstoffixation verstärkt. In den Zellen des Azotobacter fand Stocklasa bis zu $5 \% \mathrm{P}_{2} \mathrm{O}_{5}$, Hoff mann und Hammer ${ }^{2}$ ) aber 2,5 bis 3,0\%, je nach dem Alter der Kultur.

3. Die Elementaranalyse der Körper von Azotobacter ergab keine vollkommen übereinstimmenden Resultate. Wir wollen hier eine der Analysen, welche dem Mittelwerte aus den gewonnenen Zahlenergebnissen am nächsten steht, angeben:

$0,2420 \mathrm{~g}$ der Substanz ergaben $0,2047 \mathrm{~g} \mathrm{CO}_{2}$ und 0,1436 $\mathrm{g} \mathrm{H}_{2} \mathrm{O}$.

Es fanden sich also: $\mathrm{G}=22,42 \%, \mathrm{H}=6,41 \%$.

4. Bestimmung des Gesamtstickstoffs nach Kjeldahl.

Nach Gerlach und Vogel ${ }^{3}$ ) sind die Körper des Azotobacter sehr reich an Stickstoff und enthalten in getrocknetem Zustande 75-80\% Eiweiß. Einen diesem nahekommenden Wert gibt auch Stoklasa (loc. cit.) an. Ungefähr dieselben Zahlenwerte findet man auch für einige gewöhnliche Mikroben (Bac. subtilis, Sarcina lutea u. a.) verzeichnet. Bei anderen (Vibrio cholerae, Bac. pyocyaneus, Bact. coli u. a.) sind diese Zahlenwerte niedriger, sie steigen nicht über $60-65 \%$. Abweichende Resultate ergaben die Versuche von Hoffmann und Hammer (loc. cit.), welche zur Analyse Zellen des Azotobacter verwandten, die direkt von der Oberfläche des Agars gesammelt, nicht aus flüssigen Kulturen abfiltriert worden waren, wie dies in den Versuchen früherer Autoren der Fall war. In den Versuchen von Hof fmann und Hammer fand sich ein

1) Stoklasa, Centralbl. f. Bakt., 2. Abt., Bd. 21, 1908.

2) Hoffmann u. Hammer, Centralbl. f. Bakt., 2. Abt., Bd. 28, 1910.

3) Gerlach und Vogel, Centralbl. f. Bakt., 2. Abt., Bd. 9, 1902.

Hoppe-Seyler's Zeitschrift f. physiol. Chemie. LXXXVIII. 
bedeutend geringerer Gehalt an Eiweißstoffen, welcher 11 bis $18 \%$ nicht überstieg, wobei mit dem Alter der Kultur auch der Stickstoffgehalt anwuchs. Dieser Unterschied der Untersuchungsergebnisse ist dadurch $\mathrm{zu}$ erklären, daß bei Züchtung auf festen Nährböden die Zellen des Azotobacter eine voluminöse schleimige Kapsel, welche aùs stickstofffreier Substanz besteht, bilden. In flüssigen Kulturen geht ein großer Teil dieses Schleimes beim Waschen der Bakterienmasse, beim Zentrifugieren desselben usw. in Lösung über.

An jeder der 3 einzelnen Portionen von Azotobacterkörpern (A, B, C) nahmen wir je 2 Parallelbestimmungen des Stickstoffes (nach Kjehldahl) vor. Es ergaben sich folgende Resultate (berechnet wurde der N-Gehalt auf die lufttrockene Masse).

A

$\underbrace{2,18 \% \text { und } 2,17 \%}_{2,175 \%}$
B

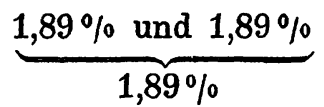

C

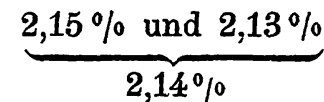

Im Durchschnitt ergaben 6 Bestimmungen 2,068\% N. Multiplizieren wir diesen Wert mit dem «Eiweißkoeffizienten * 6,25 , so erhalten wir den Eiweißgehalt im untersuchten Materiale $=12,925 \%$. Hierbei sei bemerkt, daß die Multiplikation mit dem Koeffizienten gewöhnlich ein zu hohes Fazit ergibt, da hierbei sonstige stickstoff haltige Substanzen der Zelle, z. B. die Bestandteile der Hülle, die Reservestoffe usw. nicht in Rechnung gezogen werden. Der von uns gefundene Wert nähert sich dem von Hammer und Hoffmann (loc. cit.) fesfgestellten, was auch natürlich ist, weil ja in beiden Fällen ein analoges Verfahren zum Aufsammeln der Azotobacterkörper von der Oberfläche des festen Substrates angewandt wurde. ${ }^{1}$ )

Resumieren wir die erzielten Resultate, so sehen wir, daß in der lufttrockenen Masse der Azotobacterzellen enthalten sind:

$\begin{array}{lr}\text { Wasser } & 6,63 \% \\ \text { Asche } & 4,16 \% \\ \text { Eiweißstoffe } & 12,925 \%\end{array}$

1) Es wäre vielleicht von Nutzen, hier zu erwähnen, daß bei dem anaëroben Stickstofffixator (*Butylfermento Beijerincks) $4-4,4 \% \mathrm{~N}$, d. h. 25-27,5\% Eiweiß nachgewiesen werden sinḍ. 
Die Differenz muß also auf stickstofffreie Substanz bezogen werden, deren Gehalt folglich $76,285 \%$ beträgt.

5. Die stickstofffreie Substanz.

In ihrer Hauptmasse setzt sich diese Substanz augenscheinlich aus gewucherten Hüllen der Azotobacterzellen zusammen, welche nach Beijerinck und van Delden ${ }^{1}$ ) aus Pectinkörpern bestehen. Diese letzteren gehen bekanntlich leicht in Buttersäuregärung über, worauf 'wir in bezug auf die Zellen des Azotobacter bereits hingewiesen haben. Heinze ${ }^{2}$ ) weist auf den zuweilen sehr reichlichen Gehalt an Glykogen in den Zellen des Azotobacter hin; hierdurch allein ist jedoch der überaus bedeutende Bestand an stickstofffreier Substanz, den wir in unseren Versuchen feststellen konnten, nicht zu erklären. Heinze ${ }^{3}$ ) behauptet gleichfalls, daß der Azotobacter in Gemeinschaft mit anderen Arten Kohlenwasserstoffverbindungen aus der Acetylengruppe bildet.

Nach Hoffmann ${ }^{4}$ ) bestehen die Kapseln des Azotobact er aus Kohlenhydraten, hauptsächlich aus Pentosanen und diesen nahestehenden Verbindungen. Jedoch deutet der Gehalt an Phosphor in dem Bestande der Kapseln auf eine kompliziertere chemische Zusammensetzung derselben.

In unseren Versuchen färbten sich die die Azotobacterzellen umgebenden Kapseln mit Jod nicht und reduzierten Fehlingsche Lösung nicht.

6. Hydrolyse der Azotobacterkörper. Zur Hydrolyse verwandten wir verschiedene Substanzen: Wasser, verdünnte Schwefelsäure $(1,25 \%$ und $25 \%$ und $10 \%$ ige Salzsäure. Die Versuche wurden in der Weise angestellt, daß wir eine geringe Menge des Materiales mit der entsprechenden Flüssigkeit übergossen und sodann im Laufe von 6-12 Stunden mit Rückflußkühler sieden ließen. Sodann wurde der Gewichtsverlust des hydrolysierten Materials und der Zuckergehalt im Filtrate bestimmt.

1) Beijerinck u.Van Delden, Centralbl.f. Bakt., 2.Abt., Bd.9,1902.

2) Heinze, Centralbl. f. Bakt., 2. Abt., Bd. 12, 1904.

s) Heinze, Centralbl. f. Bakt., 2. Abt., Bd. 17, 1907.

4) Hoffmann, Centralbl. f. Bakt., 2. Abt., Bd. 36, 1913. 
Bei der Hydrolyse durch Erhitzen mit Wasser konnte kein Zucker im Filtrate, wohl aber Gewichtsverlust nachgewiesen werden. In einem Falle waren über 31\% der Trockensubstanz in Lösung übergegangen. Außer den hydrolytischen Produkten gehen auch verschiedene andere Substanzen, z. B. organische Säuren, welche bei der Buttersäuregärung der Bakterienmasse entstanden waren, in Lösung über.

Bei der Hydrolyse mit 1,25\% iger Schwefelsäure und mit 10\% iger Salzsäure fanden sich im Filtrate ca. 33\% Zucker der luftrockenen Substanz.

Bei der Hydrolyse mit 25\% iger $\mathrm{H}_{2} \mathrm{SO}_{4}$ (24 Stunden auf offenem Feuer) konnten im Filtrate 10-12\% Zucker (in verschiedenen Portionen) nachgewiesen werden.

Aus diesen Befunden kann man die Schlußfolgerung machen, daß die Kapseln des Azotobacter aus irgend einem Kohlenhydrat (Polysaccharid) bestehen, welches bei Hydrolyse Zucker, der Fehlingsche Flüssigkeit reduziert, ergibt.

7. Zur Hydrolyse der Eiweißstoffe nahmen wir je $100 \mathrm{~g}$ der trockenen Bakterienmasse.

a) Hydrolyse vermittelst 25\%iger Schwefelsäure. Die trockenen Azotobacterkörper mit einem Gehalte von $2,175 \% \mathrm{~N}$ wurden mit der 10 fachen Quantität $25 \%$ iger $\mathrm{H}_{2} \mathrm{SO}_{4}$ anfangs im Laufe von 5 Stunden im Wasserbade, später 24 Stunden auf offenem Feuer mit Rückflußkühler erhitzt. Hierbei blieben 25\% der Substanz unaufgelöst. Dieser Rest wurde unter denselben Bedingungen von neuem im Laufe von 12 Stunden hydrolysiert. Danach blieben noch $22 \%$ der Bakterienmasse mit einem Gehalte von $0,755 \%$ Stickstoff $(0,1881 \mathrm{~g})$ unaufgelöst.

Der Stickstoffgehalt des Filtrates beträgt 1,7956 g, d. h. $87,33 \%$ der anfänglichen N-Menge.

Die Bestimmung des Aminostickstoffes nach Sörensen ${ }^{1}$ ) ergab $1,176 \mathrm{~g}$, d. h. amidiert wurden $54,1 \%$ des Gesamtstickstoffes oder $62 \%$ des in Lösung übergegangenen.

b) Hydrolyse mit 20\%iger Salzsäure (in einer anderen Portion des Materials, welche 2,14\% N enthielt).

1) Sörensen, Biochem. Zeitschrift, Bd. 7, 1907. 
Sämtliche Bestimmungen wurden zweimal in zwei Proben ein und desselben Materiales ( $\mathbf{a}$ und $\mathbf{b}$ ) vorgenommen. Die hydrolytischen Produkte wurden nach van Slyke ${ }^{1}$ ) bestimmt.

1. Analyse. $32,0828 \mathrm{~g}$ der Bakterienkörper wurden im Laufe von 26 Stunden mit $500 \mathrm{ccm}$ einer $20 \%$ igen Salzsäure (im Verhältnis von $1: 15$ ) hydrolysiert.

Im Hydrolysat fanden sich 0,63 g Stickstoff, d. h. 91,7\% des Gesamtstickstoffes.

\begin{tabular}{c|c|c}
\hline Es wurden bestimmt & In $\mathbf{g}$ & $\begin{array}{c}\text { In \% zum Gesamtstickstoff } \\
\text { des Hydrolysats }\end{array}$ \\
\hline Ammoniakstickstoff . . . . . & 0,06293 & 9,9 \\
Melaninstickstoff . . . . . . . & 0,0230 & 3,65 \\
Gesamtstickstoff der Diaminobasen & 0,1663 & 26,39 \\
Gesamtstickstoff der Monoamine . & 0,3780 & $\frac{60,00}{99,94}$
\end{tabular}

Vom Gesamt-N der Diamin of raktion kommen:

$$
\begin{array}{r}
\text { auf Aminostickstoff }-0,1148 \mathrm{~g}=18,22 \% \\
\text { Nichtaminostickstoff }-0,0515,=8,16 \% \\
\text { Summe } 0,1663 \mathrm{~g}=26,38 \%
\end{array}
$$

Auf folgende Diamine kommen:

$$
\begin{aligned}
& \text { Arginin }-0,0655 \mathrm{~g}=10,39 \% \\
& \text { Histidin }-0,0103 \mathrm{z}=1,63 \% \\
& \text { Lysin }-0,0914 \mathrm{*}=14,50 \% \\
& \hline 0,1672 \mathrm{~g} \quad 26,52 \%
\end{aligned}
$$

Vom Gesamt-N der Monoamine kommen:

$$
\begin{array}{r}
\text { auf Aminostickstoff }-0,3479 \mathrm{~g}=55,22 \% \\
\text { Nichtaminostickstoff }-0,0301 \%=4,779 \% \\
\text { Summe } 0,3780 \mathrm{~g} \quad 60 \%
\end{array}
$$

2. Analyse. $30 \mathrm{~g}$ der Bakterienkörper wurden im Laufe von 28 Stunden mit $300 \mathrm{ccm}$ einer $20 \%$ igen Salzsäure (1:10) hydrolysiért.

Die zur Bestimmung genommene Portion des Hydrolysates enthielt $0,5101 \mathrm{~g} \mathrm{~N}$.

7 van Slyke, Ber. d. Deutsch. chem. Ges., Bd. 43, 1910. - Biochemische Arbeitsmethoden. Bd. 5 (2. Abt.) und Bd. 6. 
W. L. Omeliansky und N. O. Sieber,

\begin{tabular}{|c|c|c|}
\hline Es wurden bestimmt & In $\mathbf{g}$ & $\begin{array}{c}\text { In } \% \text { zum Gesamtstickstoff } \\
\text { des Hydrolysats }\end{array}$ \\
\hline Ammoniakstickstoff . . . . & 0,0490 & 9,6 \\
\hline Melaninstickstoff . . . . & 0,0180 & 3,5 \\
\hline Gesamtstickstoff der Diaminobasen & 0,1355 & 26,56 \\
\hline \multirow[t]{2}{*}{ Gesamtstickstoff der Monoamine .. } & 0,3076 & 60,33 \\
\hline & 0,5101 & 99,99 \\
\hline
\end{tabular}

Vom Gesamt-N der Diaminofraktion kommen auf: Aminostickstoff Nichtaminstickstoff $0,0904 \mathrm{~g}=17,72 \%$

Summa $\overline{0,1355 \mathrm{~g}}=\overline{26,56 \%}$

Auf folgende Diamine kommen:

$\begin{array}{ll}\text { Arginin } & 0,0526 \mathrm{~g}=10,31 \% \\ \text { Histidin } & 0,008 \cdot \gg=1,64 \% \\ \text { Lysin } & 0,0745 »=14,60 \% \\ \text { Summa } & 0,1351 \mathrm{~g}=26,55 \% .\end{array}$

Vom Gesamt-N der Monoamine kommen auf:

$$
\begin{aligned}
\text { Aminostickstoff } & 0,2828 \mathrm{~g}=55,44 \% \\
\text { Nichtaminstickstoff } & 0,0248 \%=4,86 \% \\
\text { Summa } & 0,3076 »=60,30 \% .
\end{aligned}
$$

Die Cystinbestimmung ergab in beiden Analysen ein negatives Resultat. Auch Purinbasen konnten nicht nachgewiesen werden.

Fassen wir die von uns erzielten Resultate zusammen, so kommen wir zu folgenden Schlüssen:

1. Die unter den von uns eingehaltenen Bedingungen gewonnenen Zellen des Azotobacter chroococcum enthalten eine verhältnismäßig geringe Menge Eiweiß (ca. 13\%), ihre Hauptmasse besteht aus stickstofffreier Substanz (Kohlehydrate).

2. Die Verteilung des Stickstoffes von Ammoniak, Diund Monoaminen in den hydrolytischen Produkten des Azotobacter weist im Vergleiche $\mathrm{zu}$ anderen Eiweißstoffen keine Abweichungen auf, und zwar fanden wir ca. 10\% Ammoniakstickstoff, 26,5\% Diaminstickstoff und ca. 60\% Monoaminstickstoff. 
3. Einige Besonderheiten weist das Verhältnis der zu den Diaminen gehörigen Substanzen untereinander auf. Beim Azotobacter fand sich ein weit größerer Gehalt an Lysin im Vergleich zu demselben bei anderen tierischen und planzlichen Eiweißstoffen, und zwar 14,5-14,6\%. Es war sogar in größerer Quantität vertreten als wie Arginin. In den meisten Fällen ist das Verhältnis zwischen Arginin und Lysin umgekehrt (vgl. die Tabelle, welche van Slyke in Bd. 5, 2. der *Biochemischen Arbeitsmethoden», S. 1026 angibt).

4. Die Quantität des festgestellten Arginins wies keine Abweichungen von dem Mittelwerte des Gehaltes dieser Diaminosäure in den meisten Eiweißsubstanzen auf. Histidin konnte nur in geringen Mengen nachgewiesen werden.

5. Ob die erwähnten Beziehungen ein konstantes Merkmal, welches für den chemischen Bestand der Körper des Azotobacter chroococcum typisch ist, darstellen oder ob sie sich in Abhängigkeit von verschiedenen Bedingungen (dem Bestande des Nährbodens, dem Alter der Kultur, den Rasseneigenheiten usw.) verändern können, auf diese Fragen kann keine bestimmte Antwort gegeben werden. Die Inkongruenz der Untersuchungsergebnisse verschiedener Autoren, welche Analysen ein und derselben Art vorgenommen haben, spricht augenscheinlich zugunsten der zweiten Voraussetzung. 